\title{
Video on Demand \\ Over Optical Wireless Communication Links
}

\author{
M. SiRAJ ${ }^{a, *}$, M.I. MEMON ${ }^{b}$ AND M.S. KHAN ${ }^{c}$ \\ ${ }^{a}$ PSATRI, College of Engineering, King Saud University, SA \\ ${ }^{b}$ COMSATS Institute of Information Technology, Islamabad Pakistan \\ ${ }^{c}$ Department of Electrical Engineering, University College of Engineering Science and Technology, \\ Lahore Leads University, Lahore, Pakistan
}

\begin{abstract}
Video on demand technology is a modern wireless communication application which requires a large bandwidth. Optical wireless networks, as a potential alternative to radio frequency, is an emerging technology that is easier to set up and provides a cost effective solution, where a physical network is difficult to deploy. Optical wireless networks can be used as a backbone to provide the much needed bandwidth for video on demand application. In this paper, Wavelength Division Multiplexing modulation scheme is proposed for the optical wireless networks due to its significance of transmitting distinct data channels at different wavelengths. Wavelength Division Multiplexing can be easily integrated with optical wireless networks, consequently resulting in an increased data rate. This paper presents a novel approach of integrating video on demand with the state of art Wavelength Division Multiplexing based optical wireless networks. Furthermore, the paper discusses the challenges and advantages over the convention modulation schemes.
\end{abstract}

DOI: 10.12693/APhysPolA.129.826

PACS/topics: 84.40.Ua, 42.15.Eq

\section{Introduction}

The video on demand application ( $\mathrm{VoD})$ is a very popular internet service occupying $27 \%$ of the internet traffic [1]. According to a survey more than $74.1 \%$ of business and personal users access streaming media at least twice or thrice a week [2]. In the United States, 66 percent of tablet owners stream video twice or thrice or more [3]. According to a study, in United Kingdom, VoD generates $£ 200$ million revenue and another $£ 100$ million in advertisement by 2013 [4]. In 2015, VoD network traffic will be equivalent to 3 billion DVDs per month [5]. This estimated figure is much less than the present day figures. Unfortunately, we don't have the current figures. Internet $\mathrm{VoD}$ applications such as YouTube [6], PPLive [7], iPlayer [8] and Hulu [9] are very popular with the users. PPLive is a very popular VoD in China. Researchers [1016] have used this service to study VoD. As they require a high amount of bandwidth, they are very costly. Video over the internet poses some important challenges. Sometimes the network may be down or there could be congestion in the network leading to service loss. A VoD system can be configured in one of the following types, a) distributed, b) centralized and c) networked. In a distributed VoD system, the media content is stored on one central server with smaller servers around it to cater for high-demand requests. In a centralized system, the entire media content is on a centralized server. This server handles all clients' requests. In a network environment,

\footnotetext{
*corresponding author; e-mail: siraj@ksu.edu.sa
}

the media servers are spread across the network. Each server handles requests from a small group of clients only to provide an interrupted high-quality video playback. In a VoD system, the two major hurdles are high bandwidth required and the high requests associated with it. It has been a challenge for researchers to make $\mathrm{VoD}$ efficient, reliable, robust and affordable.

Free space optical (FSO) communications have gained a considerable attention due to increasing demands of higher bandwidth and nomadic application. WDM is the technology which multiplexes multiple optical carrier signals on a single medium by using different signals.

FSO links are highly dependent on weather conditions. The fog, smoke, rain and scintillations are the main challenges which significantly degrade the FSO link performance. Many techniques have been proposed to improve the performance of FSO link, like aperture increasing, using of low pass Bessel filter, wavelength of $1550 \mathrm{~nm}$ and amplification of optical signal using an optical amplifier.

Here we will study the WDM based FSO system for different atmospheric conditions.

\section{Related work}

Researchers have used wireless mesh networks (WMNs) [17] for a higher bandwidth. WMNs bandwidth was further increased by choosing appropriate routing metric [18] and decreasing interference [19]. In [20] authors presented an efficient cognitive VoD model for a higher bandwidth, whereas in [21] network connectivity was enhanced by exploiting all-optical multicast in semiconductor ring laser. 
Free-space optical links gained considerable importance for higher data rate and are potential candidates for future communication applications. FSO systems can be used to meet the broadband communication needs for both terrestrial and space applications [22]. Possible applications include satellites, deep-space probes, ground stations, unmanned aerial vehicles (UAVs), high altitude platforms (HAPs), and other nomadic communication applications. Moreover, FSO links can be used for military and civilian purposes [23].

Most commonly FSO links are based on intensity modulation and direct detection, because of their relatively simple implementation. The current trend is the implementation of wavelength division multiplexing based FSO system to achieve higher data rates $[24,25]$. Here we presented WDM based FSO system for VoD applications at the data rate of 1.25 Gbps.

\section{Proposed model}

Figure 1 below shows the block diagram of our proposed VoD model of video streaming over FSO Links.

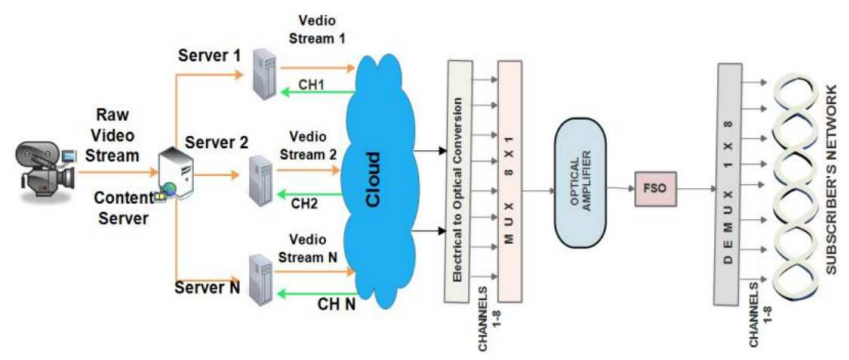

Fig. 1. Block diagram of VoD over FSO links.

The VoD model is a distributed VoD system where the media content is stored on one central server called content server with smaller servers (Server 1, Server 2, Server N, etc.) around it to cater for high demand requests. It is assumed that all the clients maintain a buffer space which keeps a record of the recent viewed popular movie (movies with high demand). As all the incoming requests vary from one client to another, they are independent of each other. Therefore, the incoming process of normal requests as well as of interactive requests to the content server can be modeled as a Poisson distribution. Whenever a client makes a request for the movie, this request goes to the content server who keeps a track of all the users. If the client is a first-time user, he has to register himself in the content server so that the list of clients watching video is maintained. The content server will guide him to the peer through which the client will be able to get the streaming video. The scheme shown in Fig. 1 has the following advantages, a) client can download from multiple sites over multipath, b) traffic load is balanced.

\section{Simulation}

Figure 1 shows the block diagram for FSO system for VoD application at the link distance of $2 \mathrm{~km}$. In the block diagram the eight channels range from $193.1 \mathrm{THz}$ to $193.8 \mathrm{THz}$ with a fixed channel spacing of $0.1 \mathrm{THz}$. They are combined together by using WDM. The system parameters are shown in Table I.

TABLE I

WDM based FSO system parameters.

\begin{tabular}{c|c}
\hline \hline Parameters & Values \\
\hline Frequency & $193.1-193.8 \mathrm{THz}$ \\
Optical amplifier type & EDFA \\
Channel spacing & $0.1 \mathrm{THz}$ \\
Bit rate & $1.25 \mathrm{Gbps}$ \\
CW laser power & $20 \mathrm{dBm}$ \\
Line width & $10 \mathrm{MHz}$ \\
Amplifier Gain & $20 \mathrm{~dB}$ \\
Link Range & $200 \mathrm{~m}$ to $2 \mathrm{~km}$ \\
Tx Aperture & $2.5 \mathrm{~cm}$ \\
Rx Aperture & $30 \mathrm{~cm}$ \\
Amplifier Noise figure & $4 \mathrm{~dB}$ \\
Beam Divergence & $2 \mathrm{mrad}$ \\
APD gain & 3 \\
Responsivity & $1 \mathrm{~A} / \mathrm{W}$ \\
Dark current & $10 \mathrm{nA}$ \\
Filter type & Low pass Bessel
\end{tabular}

The FSO link is modeled with carrier frequency of 193.1-193.8 $\mathrm{THz}$ with the channel spacing of $0.1 \mathrm{THz}$. At the transmitter end, on each channel, a $1.25 \mathrm{Gbps}$ data is generated by using a pseudorandom bit sequence generator, this data is coupled to NRZ encoder and further modulated by using Mach-Zehnder Modulator. A CW laser diode of line width of $10 \mathrm{MHz}$ with a power of $20 \mathrm{dBm}$ is used. The optical amplifier with gain of $30 \mathrm{~dB}$ is used for pre-amplification technique. At the receiver end $1 \times 8$ Demux is used and the optical signal is received by avalanche photo-detector having the dark current of $10 \mathrm{nA}$.

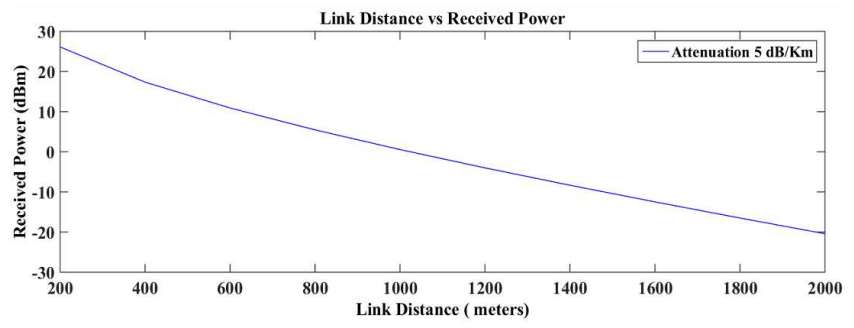

Fig. 2. Received power for WDM-FSO link operating at 2.5 Gbps with atmospheric attenuation of $5 \mathrm{~dB} / \mathrm{km}$.

As local weather conditions pose significant challenge for FSO system to get carrier class availability and reliability. Therefore, we consider the haze and light fog scenario for simulation. Haze can cause the attenuation up to $20 \mathrm{~dB} / \mathrm{km}$ and light fog can cause the attenuation up to $30 \mathrm{~dB} / \mathrm{km}$. Figures 2 and 3 show the received 
power for haze condition. Here, in both figures, the received power is shown when the attenuation is $5 \mathrm{~dB} / \mathrm{km}$ and $15 \mathrm{~dB} / \mathrm{km}$.

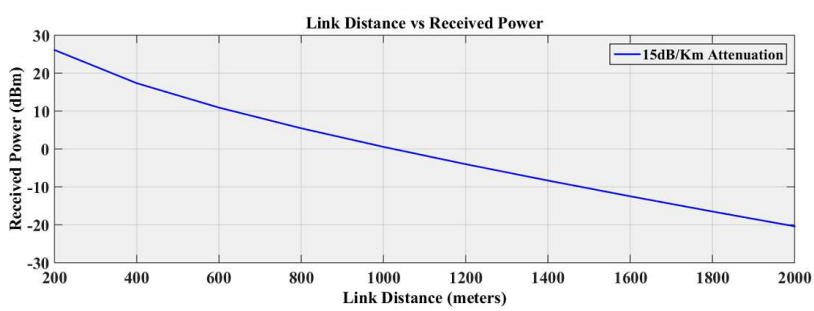

Fig. 3. Received power for WDM-FSO link operating at 1.25 Gbps with atmospheric attenuation of $15 \mathrm{~dB} / \mathrm{km}$.

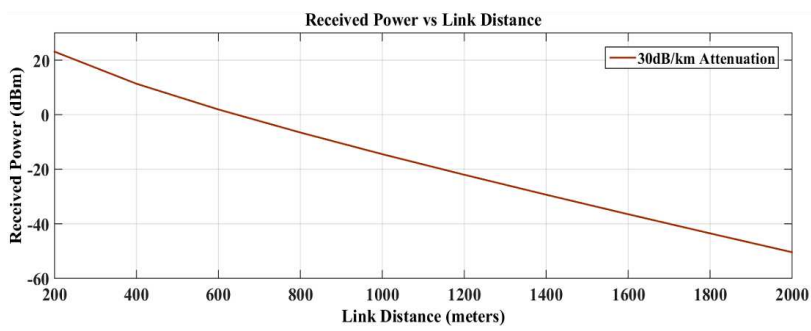

Fig. 4. Received power for WDM-FSO link operating at 1.25 Gbps with atmospheric attenuation of $30 \mathrm{~dB} / \mathrm{km}$.

Figure 4 shows the received power for WDM-FSO link operating at 1.25 Gbps with atmospheric attenuation of $30 \mathrm{~dB} / \mathrm{km}$.

\section{Conclusions}

In this study, from the simulation results it is concluded that the system can perform well to get high availability and reliability under haze and light fog conditions where attenuation may go as high as $30 \mathrm{~dB} / \mathrm{km}$. It was also observed that there was enough receiving power for signal detection.

It can be concluded from the simulation results that WDM based FSO system can be implemented up to a link distance of $2 \mathrm{~km}$ at the data rate of $1.25 \mathrm{Gbps}$ with high reliability and link availability.

\section{Acknowledgments}

The researchers wish to thank the Research Center, Faculty of Engineering, King Saud University for supporting this research.

\section{References}

[1] http://webcast.broadcastnewsroom.com/ articles/viewarticle.jsp?id=8835.

[2] http://www.streamingmedia.com, Survey (2010).
[3] http://www.streamingmedia.com/ Articles/News/Online-Video-News/ RoviSurveyShowsHighStreamingVideoRateson Mobile-Devices-87918. aspx, (2013).

[4] Cisco visual networking index: Forecast and methodology, 2010-2015. Technical report, Cisco, 2011.

[5] UK: Internet Statistics Compendium. E-consultancy, (2011).

[6] YouTube [online], available: www. youtube.com/.

[7] PPTV [online], available: www.pptv.com/.

[8] BBC iPlayer [online], available: www.bbc.co.uk/ iplayer/.

[9] Hulu [online], available: www.hulu.com/.

[10] T. Silverston, O. Fourmaux, arXiv:cs/0610133, 2006.

[11] X. Hei, C. Liang, J. Liang, Y. Liu, K.W. Ross, IEEE Trans. Multimedia 9, 1672 (2007).

[12] L. Vu, I. Gupta, J. Liang, K. Nahrstedt, Proc. QSHINE'0\%, Vancouver, BC, Canada, 2007, p. 247.

[13] X. Hei, Y. Liu, K.W. Ross, IEEE J. Sel. Areas Commun. 25, 1640 (2007).

[14] S. Spoto, R. Gaeta, M. Grangetto, M. Sereno, Proc. IPDPS'09, Rome, Italy, 2009, p. 1.

[15] P. Gao, T. Liu, Y. Chen, X. Wu, Y. El-Khatib, C. Edwards, Proc. GridNets, Springer, 2008, p. 24.

[16] Y. Huang, T.Z.J. Fu, D. Chiu, J.C.S. Lui, C. Huang, Proc. SIGCOMM'08, New York, NY, USA, 2008, p. 375.

[17] M. Siraj, K.A. Bakar, Int. J. Phys. 6, 3189 (2011).

[18] M. Siraj, K.A. Bakar, Int. J. Phys. Sci. 7, 456 (2012).

[19] M. Siraj, K.A. Bakar, J. Comput. Sci. 8, 1263 (2012).

[20] M. Siraj, Z.A. Abbasi, Computational and Business Intelligence (ISCBI), 2013 International Symposium on, 2013, p. 231.

[21] M. Siraj, M.I. Memon, M. Shoaib, S. Alshebeili, AIP Conf. Proc. 1653, 020098 (2015).

[22] M.S. Awan, R. Nebuloni, C. Capsoni, L. CsurgaiHorváth, S.S. Muhammad, F. Nadeem, M.S. Khan, E. Leitgeb, Int. J. Satell. Commun. Network. 29, 97 (2009).

[23] H. Henniger, O. Wilfert, Radio Eng. 19, 203 (2010).

[24] E. Ciaramella, Y. Arimoto, G. Contestabile, M. Presi, A. D'Errico, V. Guarino, M. Matsumoto, IEEE Photonics Technology Lett. 21, 1121 (2009).

[25] E. Ciaramella, Y. Arimoto, G. Contestabile, M. Presi, A. D'Errico, V. Guarino, M. Matsumoto, IEEE J. Selected Area 27, 1639 (2009). 